\title{
Supersymmetry and electroweak breaking from extra dimensions at the TeV scale
}

\author{
A. Delgado \\ Instituto de Estructura de la Materia (CSIC), Serrano 123, 28006 Madrid, Spain \\ A. Pomarol* \\ TH-Division, CERN, CH-1211 Geneva 23, Switzerland \\ M. Quirós \\ Instituto de Estructura de la Materia (CSIC), Serrano 123, 28006 Madrid, Spain
}

(Received 14 January 1999; published 8 October 1999)

\begin{abstract}
We analyze some features of the role that extra dimensions, of radius $R$ in the $\mathrm{TeV}^{-1}$ range, can play in the soft breaking of supersymmetry and the spontaneous breaking of electroweak symmetry. We use a minimal model where the gauge and Higgs boson sector of the MSSM are living in the bulk of five dimensions and the chiral multiplets in a four-dimensional boundary. Supersymmetry is broken in the bulk by the Scherk-Schwarz mechanism and transmitted to the boundary by radiative corrections. The particle spectrum is completely predicted as a function of a unique $R$ charge. The massless sector corresponds to the pure standard model and electroweak symmetry is radiatively broken with a light Higgs boson weighing $\lesssim 110 \mathrm{GeV}$. The $\mu$ problem is solved and Higgsinos, gauginos, and heavy Higgs bosons acquire masses $\sim 1 / R$. Chiral sfermions acquire radiative squared-masses $\sim \alpha_{i} / R^{2}$. The effective potential is explicitly computed in the bulk of extra dimensions and some cosmological consequences can be immediately drawn from it. Gauge coupling running and unification are studied in the presence of Scherk-Schwarz supersymmetry breaking. The unification is similar to that in the supersymmetric theory. [S0556-2821(99)04621-4]

PACS number(s): 11.30.Pb, 04.50.+h, 11.10.Kk, 12.60.Jv
\end{abstract}

\section{INTRODUCTION}

The standard model (SM) of strong and electroweak interactions is being tested by present high-energy colliders for energies $\lesssim 200 \mathrm{GeV}$ and is proving to describe the corresponding interactions with great accuracy. By the same token those experiments are putting limits on the scale of new physics, suggesting thus that extra matter, if it exists, is only relevant in the $\mathrm{TeV}$ scale range. In particular these limits do apply to the best motivated of the phenomenological lowenergy extensions of the SM, its minimal supersymmetric extension (MSSM), putting bounds around the TeV scale on the mass of the supersymmetric partners. On the other hand, the origin of the electroweak symmetry breaking (EWSB) mechanism remains as the missing building block of the SM, or any low energy extension thereof, given the fact that the Higgs boson field has been shown so elusive in all experiments to the present day. In the context of the MSSM the problems of the origin and stability of the EWSB scale are alleviated because the latter is related to the scale of supersymmetry breaking, and it is further protected against radiative corrections by supersymmetry: the sensitivity of the Higgs boson squared-mass to the high scale is not quadratic (as in the case of the SM) but only logarithmic. However the origin of supersymmetry breaking remains the big unsolved problem in these theories.

The presence of supersymmetry in the low-energy extension of the SM is further supported by the fact that consistent

\footnotetext{
*On leave from IFAE, Universitat Autònoma de Barcelona, E-08193 Bellaterra, Barcelona.
}

fundamental theories aiming to unify gauge and gravitational interactions at high scales (string theories) are supersymmetric. A common feature of these theories is the presence of (compactified) extra dimensions. If all dimensions are small, of the order of the Planck or grand unified theory (GUT) length, their detectability is outside the scope of present or future accelerators. However, if some radii are larger, they might have a number of theoretical and phenomenological implications [1-4].

From the fundamental point of view the presence of large extra dimensions has been proved to be essential to describe the strong coupling regime of certain string theories [5], while TeV scale superstrings have been constructed [6-8]. From the more phenomenological point of view the presence of large dimensions can play a prominent role for gauge coupling unification [9-12], for neutrino mass generation [13], to provide possible alternative solutions to the hierarchy problem [14-18], and as a transmitter of supersymmetry breaking between different boundaries [19]. On the other hand theories with a TeV higher dimensional Planck scale predict modifications of gravitational measurements in the submillimeter range [20], while some features of TeV scale quantum gravity theories have recently been worked out [21]. Moreover, the usual problems which arise in any fundamental theory, as, e.g., the proton stability and flavor conservation problems, become more acute when the fundamental (string) scale is hierarchically below the Planck scale. The most natural solution to those problems usually arises from the presence of some discrete gauge symmetries $\left(\mathbb{Z}_{N}\right)$ in the fundamental (string) theory, that are protected against gravitational radiative corrections and can suppress dangerous higher dimensional operators corresponding to proton decay 
and flavor changing neutral interactions. Finally experimental detection of $\mathrm{TeV}$ radii in future accelerators has been proposed as an unambiguous signature of large extra dimensions $[22,23]$.

In the above theoretical constructions, where compactification scales are in the $\mathrm{TeV}$ range, it is therefore tempting to assume that both the compactification scale of large dimensions and the scale of supersymmetry breaking have a common origin. This is the case if supersymmetry is broken by a continuous compactification along the compact dimension by means of the so-called Scherk-Schwarz (SS) mechanism $[24,25]$. The SS mechanism has been recently used to break supersymmetry in sectors with which we only share gravitational $[27,28]$ interactions (gravity mediated scenarios) and also in sectors that share gauge interactions with the observable sector [29-32] (gauge mediated scenarios).

In this paper we will restrict ourselves to the latter scenarios, where both the compactification and the supersymmetry breaking scales are in the $\mathrm{TeV}$ range and can then leave a characteristic signature in the present or next generation of high-energy colliders: these scenarios will in this way be testable in the near future. In particular we will concentrate in simple five-dimensional (5D) models where SS compactification acts on the fifth dimension, as those presented in Refs. [30,32], where the main features of electroweak and supersymmetry breaking already appear. In this sense our approach will be a bottom-up one, but keeping in mind that it might possibly appear in compactifications of some more fundamental higher dimensional theory. However, as we will see the resulting low energy theory will show very little sensitivity to the physics at the high scale (cutoff).

The plan of this paper is as follows. In Sec. II we will present the simplest MSSM extension in 5D, compactified on $S^{1} / Z_{2}$, along the lines of the model analyzed in Refs. $[30,32]$. All nonchiral matter (the gauge and Higgs boson sectors) will be placed on the bulk of the fifth dimension while chiral matter (chiral fermion supermultiplets) live on the $4 \mathrm{D}$ boundaries. The corresponding 5D and 4D tree-level Lagrangians and their interactions are explicitly written in Secs. II A and II B, including contact interactions, between fields in the bulk and the boundary, which are necessary for the consistency of the theory. Supersymmetry is broken by the SS mechanism in the bulk, using the $\mathrm{U}(1)_{R} R$ symmetry of the $N=2$ supersymmetry algebra. The resulting massless spectrum contains just the SM particle content plus the sfermions living on the boundary that do not have excitations along the fifth dimension and thus do not receive any mass from the SS mechanism. One-loop radiative corrections will be studied in Sec. III for the bulk, Sec. III A, and the boundary, Sec. III B. In the bulk the one-loop effective potential, in the background of the Higgs boson field zero mode, is computed and found to have a closed analytic form in terms of polylogarithm functions. The Higgs boson squared-mass at the origin is computed by diagrammatic methods and shown to agree with that obtained from the effective potential: it is positive definite in the minimal MSSM extension, which asks for radiative corrections on the boundary to trigger electroweak symmetry breaking (a common procedure in theories where supersymmetry breaking is gauge mediated to the squark and slepton sector). Radiative corrections on the boundary are computed in Sec. III B by using diagrammatic methods. Supersymmetry breaking is mediated by gauge and Yukawa interactions from the bulk to the boundary. Explicit expressions are given for soft-breaking terms. In particular soft masses for sfermions and trilinear soft-breaking couplings are computed. Because of the presence of the fifth dimension the breaking is extremely soft and does not depend at all on the details of the ultraviolet physics. The soft masses can then be predicted as a function of a unique $R$ charge. Electroweak symmetry breaking is analyzed in Sec. IV. It is triggered (as in gauge mediation) by two-loop corrections induced on the Higgs boson mass at the origin from sfermion soft masses on the boundary. These corrections are numerically relevant, due to the smallness of the bulkgenerated positive Higgs boson squared-mass, and must be the leading two-loop corrections to the effective potential. Minimization of the whole effective potential leads to electroweak symmetry breaking at the correct scale with a light SM-like Higgs boson and very heavy supersymmetric particles. The rough features of the mass spectrum are as follows: the Higgs boson mass is bounded by $\lesssim 110 \mathrm{GeV}$, squarks and sleptons have masses $\sim 1 \mathrm{TeV}$ while heavy Higgs bosons, gauginos and Higgsinos weigh $\sim 10 \mathrm{TeV}$. The model does not have any $\mu$ problem in the sense that the SS mechanism provides an effective $\mu$ parameter $\sim 1 / R$. It predicts a light Higgs boson, which means that it can be probed at the CERN $e^{+} e^{-}$collider LEP, and a right-handed slepton as the lightest supersymmetric particle (LSP) which can generate a cosmological problem unless $R$ parity is broken or there are light right-handed neutrinos $[32,33]$. Other alternative models with heavier Higgs bosons and a neutralino as LSP have been discussed in Sec. V. The most obvious possibility is having the chiral and gauge sector living in the bulk of the fifth dimension and the Higgs boson sector on the boundaries. These models suffer from the $\mu$ problem and present some experimental peculiarities. Finally the issue of unification in the presence of SS supersymmetry breaking has been studied in Sec. VI. We have shown that gauge coupling running for the theory with SS supersymmetry breaking proceeds as in supersymmetric theories. In particular we have shown that the dependence of the gauge coupling running on the SS breaking parameters is extremely tiny, and corresponds to one part in $10^{7}$. Section VII contains our conclusions and Appendixes $\mathrm{A}$ and $\mathrm{B}$ are devoted to present some technical details corresponding to the problem of gauge fixing in the 5D theory and the calculation of the effective potential.

\section{TREE-LEVEL LAGRANGIAN}

In this section we present a simple model based on an $N=15 \mathrm{D}$ theory compactified on $S^{1} / Z_{2}$. The gauge sector and the nonchiral matter live in the bulk of the fifth dimension. In the minimal model the nonchiral matter consists on the Higgs boson sector. In nonminimal models we could add any vectorlike extra matter, as, e.g., $\mathrm{SU}(2)_{L}$ triplets or complete $S U(5)$ representations plus their antiparticles. In what follows we will restrict ourselves to the minimal model. $N$ 
TABLE I. Complete $N=1$ towers of states in the model.

\begin{tabular}{lccccc}
\hline \hline \multirow{2}{*}{$N=1$} & zero modes & \multicolumn{2}{c}{$N=2$ nonzero $(n>0)$ modes } \\
Vector & Chiral & Vector & \multicolumn{2}{c}{ Hyper } \\
\hline$V_{\mu}^{(0)}$ & $H_{2}^{2(0)}$ & $H_{1}^{1(0)}$ & $V_{\mu}^{(n)} ; \Sigma^{(n)}, V_{5}^{(n)}$ & $H_{1}^{1(n)} ; H_{2}^{1(n)}$ & $H_{2}^{2(n)} ; H_{1}^{2(n)}$ \\
$\lambda_{L}^{1(0)}$ & $\Psi_{L}^{2(0)}$ & $\Psi_{R}^{1(0)}$ & $\lambda_{L}^{1(n)} ; \lambda_{L}^{2(n)}$ & $\Psi_{R}^{1(n)} ; \Psi_{L}^{1(n)}$ & $\Psi_{L}^{2(n)} ; \Psi_{R}^{2(n)}$ \\
\hline \hline
\end{tabular}

$=14 \mathrm{D}$ chiral multiplets, with well defined renormalizable interactions with themselves and with the bulk fields, live on the $4 \mathrm{D}$ boundaries (fixed points of the orbifold $S^{1} / \mathbb{Z}_{2}$ ). The reason for having the chiral matter on the boundary is not chirality, that can be obtained by the $\mathbb{Z}_{2}$ projection, but the fact that chiral fermions in the bulk would not receive any mass from the electroweak breaking mechanism due to the underlying $N=2$ 4D symmetry of the Lagrangian.

\section{A. Fields in the 5D bulk}

The gauge fields in the 5D bulk belong to the vector supermultiplet $\mathrm{V}=g \mathrm{~V}^{\alpha} T^{\alpha}$, where $T^{\alpha}$ are the generators in the adjoint representation of the gauge group $\mathrm{SU}(3) \times \mathrm{SU}(2)_{L}$ $\times \mathrm{U}(1)_{Y}$ and $g$ the corresponding gauge couplings. The onshell field content of $\mathrm{V}$ is $\mathrm{V}=\left(V_{M}, \lambda^{i}, \Sigma\right)$, where $M$ $=\mu, 5$ ( $\mu$ is the 4D index), $\lambda^{i}$ is a simplectic-Majorana spinor whose superindex $i=1,2$ transforms as a doublet of the $\mathrm{SU}(2)_{R} R$ symmetry and $\Sigma$ is a real scalar. The Higgs boson fields belong to the hypermultiplets $\mathbb{H}^{a}$ [an $\mathrm{SU}(2)_{L}$ doublet with hypercharge $Y=-1 / 2]$ whose superindex $a$ $=1,2$ transforms as the doublet of a global group $\mathrm{SU}(2)_{H}$. The field content, on-shell, of the Higgs boson hypermultiplets is $\mathbb{H}^{a}=\left(H_{i}^{a}, \Psi^{a}\right)$, where $H_{i}^{a}$ are complex Higgs boson doublets and $\Psi^{a}$ are Dirac spinors.

The 5D Lagrangian for vector and hypermultiplets is given by $[30,34]$

$$
\begin{aligned}
\mathcal{L}_{5}= & \operatorname{Tr} \frac{1}{g^{2}}\left\{-\frac{1}{2} F_{M, N}^{2}+\left|D_{M} \Sigma\right| 2+i \lambda_{i} \gamma^{M} D_{M} \lambda_{i}-\bar{\lambda}_{i}\left[\Sigma, \lambda^{i}\right]\right\} \\
& +\left|D_{M} H_{i}^{a}\right|^{2}+i \bar{\Psi}_{a} \gamma^{M} D_{M} \Psi^{a}-\left(i \sqrt{2} H_{a}^{\dagger i} \bar{\lambda}_{i} \Psi^{a}+\text { H.c. }\right) \\
& -\bar{\Psi}_{a} \Sigma \Psi^{a}-H_{a}^{\dagger i} \Sigma^{2} H_{i}^{a}-\frac{g^{2}}{2} \sum_{m, \alpha}\left[H_{a}^{\dagger i}\left(\sigma^{m}\right)_{i}^{j} T^{\alpha} H_{j}^{a}\right]^{2},
\end{aligned}
$$

where the $\mathrm{SU}(2)_{R} \times \mathrm{SU}(2)_{H}$ invariance is explicit. Upon compactification of the theory on $S^{1} / Z_{2}$ the $5 \mathrm{D}$ fields are classified under the $\mathbb{Z}_{2}$ parity into even and odd fields. The even fields are the vector multiplet $\left(V_{\mu}, \lambda_{L}^{1}\right)$ and the chiral multiplets $\left(H_{2}^{2}, \Psi_{L}^{2}\right)$ and $\left(H_{1}^{1}, \Psi_{R}^{1}\right)$. The odd fields are the chiral multiplets $\left(V_{5}, \Sigma, \lambda_{L}^{2}\right),\left(H_{1}^{2}, \Psi_{R}^{2}\right)$, and $\left(H_{2}^{1}, \Psi_{L}^{1}\right)$. The $Z_{2}$ parity projects out half of the states. In fact the odd component of the zero modes is projected away while the net number of towers is divided by 2 since the positive nonzero modes of odd fields replace the negative nonzero modes of even fields. In this way the zero modes have an $N=1$ supersymmetry while the nonzero modes are arranged into $N=2$ multiplets in the way that can be seen in Table I.
We have separated by a semicolon the nonzero modes coming from an even 5D field (on the left hand side) from those coming from an odd 5D field (on the right hand side). We can then see in Table I how the complete $(n=$ $-\infty, \ldots,+\infty)$ towers are constituted.

As we can see from Table I the massless sector of the theory coincides with the MSSM. The zero modes of the gauge and Higgs boson 5D fields are 4D fields with $N=1$ supersymmetric interactions, while the chiral fields are required to live on the boundaries and so they are genuine 4D $N=1$ fields, as we will see in the next section, and they complete, along with the zero modes of the bulk fields, the MSSM. Of course, to agree with experimental data supersymmetry has to be broken. The Scherk-Schwarz (SS) mechanism [24] was used in Ref. [30] to break supersymmetry by means of a $\mathrm{U}(1)_{R} \times \mathrm{U}(1)_{H}$ global symmetry of the theory [a subgroup of the previously mentioned $\mathrm{SU}(2)_{R}$ $\times \mathrm{SU}(2)_{H}$ group] with the corresponding charges $\left(q_{R}, q_{H}\right),{ }^{1}$ which the mass spectrum depends upon. ${ }^{2}$

After the SS supersymmetry breaking the $n$-KK mass eigenstates $(n>0)$ are now given by two Majorana fermions (gauginos) $\lambda^{( \pm n)}$, two Dirac fermions (Higgsinos) $\widetilde{H}^{( \pm n)}$ and four scalar bosons (Higgs boson) $h^{( \pm n)}$ and $H^{( \pm n)}$, defined by

$$
\begin{aligned}
& \lambda^{( \pm n)} \equiv\left(\lambda_{L}^{1(n)} \pm \lambda_{L}^{2(n)}\right) / \sqrt{2}, \\
& \widetilde{H}^{( \pm n)} \equiv\left(\Psi^{1(n)} \pm \Psi^{2(n)}\right) / \sqrt{2}, \\
& h^{( \pm n)} \equiv\left[H_{1}^{1(n)}+H_{2}^{2(n)} \mp\left(H_{2}^{1(n)}-H_{1}^{2(n)}\right)\right] / 2, \\
& H^{( \pm n)} \equiv\left[H_{1}^{1(n)}-H_{2}^{2(n)} \mp\left(H_{2}^{1(n)}+H_{1}^{2(n)}\right)\right] / 2 .
\end{aligned}
$$

The corresponding masses are given in Table II, where now $n \in \mathbb{Z}$ runs over a whole tower. The masses of the zero modes of the even 5D Higgs bosons

$$
\begin{aligned}
& h^{(0)}=\frac{1}{\sqrt{2}}\left(H_{1}^{1(0)}+H_{2}^{2(0)}\right), \\
& H^{(0)}=\frac{1}{\sqrt{2}}\left(H_{1}^{1(0)}-H_{2}^{2(0)}\right),
\end{aligned}
$$

\footnotetext{
${ }^{1}$ The SS mechanism has deep roots in supergravity [24] and superstring [25] theories, where it is known to break spontaneously local supersymmetry. Had we included gravity, the 4D $N=1$ supergravity constituted by the zero modes would be spontaneously broken with a gravitino mass $m_{3 / 2}=q_{R} / R$, where $R$ is the fifth dimension radius. For other ideas of breaking supersymmetry in a $5 \mathrm{D}$ theory see Ref. [26].

${ }^{2}$ Strictly speaking only the $R$ symmetry $\mathrm{U}(1)_{R}$ breaks supersymmetry, while $\mathrm{U}(1)_{H}$ generates a common (supersymmetric) mass shift for bosons and fermions. This can be explicitly seen from the mass spectrum of Table II: had we taken $q_{R}=0$ the corresponding mass spectrum would be supersymmetric.
} 
TABLE II. Mass eigenvalues of KK states.

\begin{tabular}{ccc}
\hline \hline Field & Mass \\
\hline$\lambda^{(n)}$ & $\frac{\left|n+q_{R}\right|}{R}$ \\
$\widetilde{H}^{(n)}$ & $\frac{\left|n+q_{H}\right|}{R}$ \\
$h^{(n)}$ & $\frac{\left|n+\left(q_{R}-q_{H}\right)\right|}{R}$ \\
$H^{(n)}$ & $\frac{\left|n+\left(q_{R}+q_{H}\right)\right|}{R}$ \\
\hline
\end{tabular}

are given by $\left(q_{R}-q_{H}\right) / R$ and $\left(q_{R}+q_{H}\right) / R$, respectively. In the generic case $q_{R} \neq q_{H}$ no massless zero mode is left in the theory and this prevents electroweak symmetry breaking (EWSB). In the particular case $q_{R}=q_{H} \equiv \omega$ the Higgs doublet $h^{(0)}$ is massless, the Higgs sector coincides with that of the standard model and EWSB can proceed by radiative corrections, as we will discuss in Sec. IV where we will focus on this case. In the limiting case $\omega=1 / 2$ there is an extra massless mode, $H^{(-1)}$ and the Higgs boson sector is identical to that of the MSSM. This case will also be discussed in Sec. IV where we will show that it is phenomenologically unappealing due to the fact that the down fermion sector remains massless since the Higgs that couple to it do not get a vaccum expectation value (VEV).

\section{B. Fields on the 4D boundary}

When considering a 5D theory in an orbifold $S^{1} / Z_{2}$ coupled to two 4D boundaries, one has to be careful when dealing with the off-shell formulation of 5D supermultiplets. The reason being, as discussed in Ref. [19], that on top of the even 5D fields, also the $\partial_{5}$ of odd 5D fields couple to the boundary, since they are part of the auxiliary fields of the corresponding $N=1$ supersymmetry algebra.

In the case of a vector multiplet in the bulk, the off-shell multiplet is obtained by adding an $\mathrm{SU}(2)_{R}$ triplet of realvalued auxiliary fields $X^{A}$ [34]: $V=\left(V_{M}, \lambda^{i}, \Sigma, X^{A}\right)$. Classification under $Z_{2}$ yields even $\left(V_{\mu}, \lambda_{L}^{1}, X^{3}\right)$ and odd $\left(V_{5}, \Sigma, \lambda_{L}^{2}, X^{1,2}\right)$ vector superfields. On the boundary, it was shown in Ref. [19], that the off-shell multiplet $\left(V_{\mu}, \lambda_{L}^{1}, D\right)$, with

$$
D=X^{3}-\partial_{5} \Sigma
$$

closes the $N=1$ supersymmetry algebra. The reason for that can be traced from the transformation law of the particular combination (2.4): it transforms as a total derivative under a supersymmetry transformation, which is precisely what it is expected for a $D$ field.

The same argument is valid in the case of a hypermultip- let in the bulk. ${ }^{3}$ The even auxiliary field gets mixed with the odd field of the 5D hypermultiplet. In particular, the off-shell Higgs boson hypermultiplet is given by $\mathbb{H}^{a}=\left(H_{i}^{a}, \Psi^{a}, F_{i}^{a}\right)$, which splits into even, $\left(H_{2}^{2}, \Psi_{L}^{2}, F_{2}^{2}\right),\left(H_{1}^{1}, \Psi_{R}^{1}, F_{1}^{1}\right)$, and odd, $\left(H_{1}^{2}, \Psi_{R}^{2}, F_{1}^{2}\right),\left(H_{2}^{1}, \Psi_{L}^{1}, F_{2}^{1}\right)$, chiral superfields. On the boundary, the off-shell chiral supermultiplets are

$$
\begin{aligned}
& \mathcal{H}_{2}=\left(H_{2}^{2}, \Psi_{L}^{2}, F_{2}\right), \\
& \mathcal{H}_{1}=\left(H_{1}^{1 \dagger}, \bar{\Psi}_{R}^{1}, F_{1}^{\dagger}\right),
\end{aligned}
$$

where the corresponding $F$ fields are given by

$$
\begin{aligned}
& F_{2}=F_{2}^{2}-\partial_{5} H_{1}^{2}, \\
& F_{1}=F_{1}^{1}-\partial_{5} H_{2}^{1} .
\end{aligned}
$$

The auxiliary $F$ and $D$ fields appear now in the 4D Lagrangian of the boundary fields as in a normal $N=1 \mathrm{La}-$ grangian. When those fields are integrated out by means of their equations of motions, new interactions terms do appear. We will now use those results in our particular model, the full development can be seen in Ref. [19].

Let us consider a family of left- and right-handed quark superfields $\left(\widetilde{Q}, q_{L}\right),\left(\widetilde{U}, u_{R}\right)$ and $\left(\widetilde{D}, d_{R}\right)$ living on the boundary at $x_{5}=0 .{ }^{4}$ Since only the $5 \mathrm{D}$ even fields are nonvanishing at $x_{5}=0$, the gauge and Higgs boson supermultiplets form $N=1$ supermultiplets on the boundary.

The gauge superfield, $\left(V_{\mu}, \lambda_{L}^{1}, X^{3}-\partial_{5} \Sigma\right)$, couplings to the left-handed quark superfields are given in Refs. [35,19]. After eliminating the auxiliary field $X^{3}$, we get ${ }^{5}$

$$
\begin{aligned}
\mathcal{L}_{5}= & {\left[\left|D_{\mu} \widetilde{Q}\right|^{2}+i \bar{q}_{L} \sigma^{\mu} D_{\mu} q_{L}-\sqrt{2} i\left(\widetilde{Q}^{\dagger} \lambda_{L}^{1} q_{L}+\text { H.c. }\right)\right.} \\
& -\widetilde{Q}^{\dagger}\left(\partial_{5} \Sigma\right) \widetilde{Q}-\frac{g^{2}}{2} \sum_{\alpha}\left(\widetilde{Q}^{\dagger} T^{\alpha} \widetilde{Q}\right)^{2} \delta\left(x_{5}\right) \\
& \left.-g^{2} \sum_{\alpha}\left(\widetilde{Q}^{\dagger} T^{\alpha} \widetilde{Q}\right)\left(H_{a}^{\dagger i}\left(\sigma^{3}\right)_{i}^{j} T^{\alpha} H_{j}^{a}\right)\right] \delta\left(x_{5}\right) .
\end{aligned}
$$

\footnotetext{
${ }^{3}$ In this case two different auxiliary fields are needed because of the $\mathrm{SU}(2)$ automorphism group of the supersymmetry algebra.

${ }^{4}$ The choice of the boundary $x_{5}=0$ is completely general since we can always make a change of variables in the orbifold $x_{5}^{\prime}=x_{5}$ $-\pi R$ interchanging both boundaries. Our only hypothesis is that all chiral matter is located on the same boundary. Models with matter on both boundaries would give rise to a different phenomenology.

${ }^{5}$ The last term in Eq. (2.7) comes from the interaction $H_{a}^{\dagger i}\left(\sigma^{3}\right)_{i}^{j} X^{3} H_{j}^{a}$ in the off-shell formulation of the 5D Lagrangian. Notice that this term was absent from Ref. [19] because only gauge fields were supposed to live in the bulk therein. It will appear in general whenever there is matter in the bulk and in the boundary with common gauge interactions. In our case no such term will appear for $\mathrm{SU}(3)_{c}$, while terms mixing $\widetilde{Q}, \widetilde{U}, \widetilde{D}, \widetilde{L}$, and $\widetilde{E}$ in the boundary with the Higgs boson sector in the bulk, as in Eq. (2.7), will appear for the $\mathrm{SU}(2)_{L}$ and $\mathrm{U}(1)_{Y}$ gauge groups.
} 
A similar expression to Eq. (2.7) holds for the couplings to the right-handed superfields. After reducing the Lagrangian (2.7) to $4 \mathrm{D}$, one has in the physical basis

$$
\begin{aligned}
\mathcal{L}_{4}= & \left|D_{\mu} \widetilde{Q}\right|^{2}+i \bar{q}_{L} \sigma^{\mu} D_{\mu} q_{L}-\sum_{n=-\infty}^{\infty} \sqrt{2} i\left(\widetilde{Q}^{\dagger} \lambda^{(n)} q_{L}+\text { H.c. }\right) \\
& -\sqrt{2} \sum_{n=1}^{\infty} \frac{n}{R} \widetilde{Q}^{\dagger} \Sigma^{(n)} \widetilde{Q}-\frac{g^{2} \pi R}{2} \sum_{\alpha}\left(\widetilde{Q}^{\dagger} T^{\alpha} \widetilde{Q}\right)^{2} \delta(0) \\
& -\frac{g^{2}}{2} \sum_{n=-\infty}^{\infty} \sum_{\alpha} \widetilde{Q}^{\dagger} T^{\alpha} \widetilde{Q}\left(h^{(n)} T^{\alpha} H^{(n) \dagger}+\text { H.c. }\right),
\end{aligned}
$$

where

$$
\begin{aligned}
D_{\mu} & =\partial_{\mu}+i V_{\mu}^{(0)}+i \sqrt{2} \sum_{n=1}^{\infty} V_{\mu}^{(n)}, \\
\delta(0) & =\frac{1}{\pi R} \sum_{n=-\infty}^{\infty} 1,
\end{aligned}
$$

and $g$ is now the $4 \mathrm{D}$ gauge coupling that is related to the $5 \mathrm{D}$ gauge coupling by $\left.g\right|_{4 \mathrm{D}}=\left.g\right|_{5 \mathrm{D}} / \sqrt{\pi R}$.

The Yukawa couplings of the Higgs boson chiral superfield $\mathcal{H}_{2}$ to the quark superfields on the boundary are given by

$$
\begin{aligned}
\mathcal{L}_{5}= & \left\{h_{t}\left[H_{2}^{2} q_{L} u_{R}+\Psi_{L}^{2}\left(\widetilde{Q} u_{R}+q_{L} \widetilde{U}\right)-\left(\partial_{5} H_{1}^{2}\right) \widetilde{Q} \widetilde{U}+\text { H.c. }\right]\right. \\
& \left.-\left|h_{t} H_{2}^{2} \widetilde{Q}\right|^{2}-\left|h_{t} H_{2}^{2} \widetilde{U}\right|^{2}-\left|h_{t} \widetilde{U} \widetilde{Q}\right|^{2} \delta\left(x_{5}\right)\right\} \delta\left(x_{5}\right),
\end{aligned}
$$

and a similar expression holds for the coupling of the Higgs boson multiplet $\mathcal{H}_{1}$ to the quark superfields on the boundary with the Yukawa coupling $h_{b}$. After reduction to $4 \mathrm{D}$, the Lagrangian in Eq. (2.10) yields, in the physical basis,

$$
\begin{aligned}
\mathcal{L}_{4}= & \sum_{n=-\infty}^{\infty}\left[\frac{h_{t}}{\sqrt{2}}\left(h^{(n)}-H^{(n)}\right) q_{L} u_{R}\right. \\
& +h_{t} \widetilde{H}_{L}^{(n)}\left(\widetilde{Q} u_{R}+q_{L} \widetilde{U}\right)+\text { H.c. } \\
& -\frac{h_{t}^{2}}{2}\left[\left|\left(h^{(n)}-H^{(n)}\right) \widetilde{Q}\right|^{2}+\left|\left(h^{(n)}-H^{(n)}\right) \widetilde{U}\right|^{2}\right. \\
& \left.+|\widetilde{Q} \widetilde{U}|^{2}\right]-h_{t} \frac{n+q_{R}-q_{H}}{\sqrt{2} R} h^{(n)} \widetilde{Q} \widetilde{U} \\
& \left.+h_{t} \frac{n+q_{R}+q_{H}}{\sqrt{2} R} H^{(n)} \widetilde{Q} \widetilde{U}+\text { H.c. }\right] .
\end{aligned}
$$

For the bottom sector one obtains

$$
\begin{aligned}
\mathcal{L}_{4}= & \sum_{n=-\infty}^{\infty}\left[\frac{h_{b}}{\sqrt{2}}\left(h^{(n)}+H^{(n)}\right)^{\dagger} q_{L} d_{R}\right. \\
& +h_{b} \widetilde{\widetilde{H}}_{R}^{(n)}\left(\widetilde{Q} d_{R}+q_{L} \widetilde{D}\right)+\text { H.c. } \\
& -\frac{h_{b}^{2}}{2}\left[\left|\left(h^{(n)}+H^{(n)}\right) \widetilde{Q}\right|^{2}+\left|\left(h^{(n)}+H^{(n)}\right) \widetilde{D}\right|^{2}\right. \\
& \left.+|\widetilde{Q} \widetilde{D}|^{2}\right]+h_{b} \frac{n+q_{R}-q_{H}}{\sqrt{2} R} h^{(n) \dagger} \widetilde{Q} \widetilde{D} \\
& \left.+h_{b} \frac{n+q_{R}+q_{H}}{\sqrt{2} R} H^{(n) \dagger} \widetilde{Q} \widetilde{D}+\text { H.c. }\right] .
\end{aligned}
$$

\section{ONE-LOOP CORRECTIONS}

In the previous section we have depicted the tree-level structure of the model. Upon compactification to 4D on $S^{1} / Z_{2}$ and supersymmetry breaking by the SS mechanism, the mass spectrum and couplings of zero-modes and KK excitations depend on two parameters $q_{R}$ and $q_{H}$. Even if supersymmetry is broken in the bulk, for $q_{R}=q_{H}=\omega$ there is a massless Higgs boson doublet $h^{(0)}$, and EWSB should proceed by radiative corrections. ${ }^{6}$ To achieve this task we have to compute the Higgs boson mass induced by one-loop radiative corrections in the bulk or, more generally, the oneloop effective potential in the bulk in the presence of a constant background Higgs boson field. On the other hand, supersymmetry, though broken in the bulk by the SS mechanism, is unbroken on the boundary. Transmission of supersymmetry breaking from the bulk to the boundary should proceed by radiative corrections as we will see in this section. This transmission will be gauge mediated and thus free of any problem related to flavor changing neutral currents. These issues will be studied in the present section.

\section{A. Radiative corrections in the 5D bulk}

We will start by considering the effect of a tower of KK states with different masses for bosons and fermions

$$
\begin{gathered}
m_{B}^{2}=\left(n+q_{B}\right)^{2} \frac{1}{R^{2}}, \\
m_{F}^{2}=\left(n+q_{F}\right)^{2} \frac{1}{R^{2}}, \quad n=0, \pm 1, \pm 2, \ldots .
\end{gathered}
$$

We want to compute the one-loop effective potential for a massless scalar mode $\phi$ (the one to be associated with the SM Higgs boson field $h^{(0)}$ ), induced by this tower of KK states. This is given in the Landau gauge (see Appendix A) by

\footnotetext{
${ }^{6}$ For the case $q_{R} \neq q_{H}$ all Higgs boson doublets acquire a tree level mass $\sim 1 / R$, which would prevent the possibility of EWSB for values of $1 / R$ in the $\mathrm{TeV}$ range.
} 


$$
V=\frac{1}{2} \operatorname{Tr} \int \frac{d^{4} p}{(2 \pi)^{4}} \sum_{n=-\infty}^{\infty} \ln \left(\frac{p^{2}+M^{2}(\phi)+\left(n+q_{B}\right)^{2} / R^{2}}{p^{2}+M^{2}(\phi)+\left(n+q_{F}\right)^{2} / R^{2}}\right),
$$

where $\mathrm{Tr}$ is the trace over the number of degrees of freedom of the KK tower and $M^{2}(\phi)$ is the $\phi$-dependent mass of the KK-states. In Eq. (3.2) we must first perform the summation over the KK states and then integrate with respect to the momentum. The calculation has been performed in Appendix B using techniques borrowed from finite temperature calculations. We obtain

$$
V=\frac{1}{128 \pi^{6} R^{4}} \operatorname{Tr}\left[V\left(r_{F}, \phi\right)-V\left(r_{B}, \phi\right)\right]
$$

where

$$
\begin{aligned}
V\left(r_{i}, \phi\right)= & x^{2} \operatorname{Li}_{3}\left(r_{i} e^{-x}\right)+3 x \mathrm{Li}_{4}\left(r_{i} e^{-x}\right)+3 \mathrm{Li}_{5}\left(r_{i} e^{-x}\right) \\
& + \text { H.c., } \\
& x=2 \pi R \sqrt{M^{2}(\phi)}, \quad r_{i}=e^{i 2 \pi q_{i}}
\end{aligned}
$$

and $\mathrm{Li}_{n}(x)$ are the polylogarithm functions

$$
\operatorname{Li}_{n}(x)=\sum_{k=1}^{\infty} \frac{x^{k}}{k^{n}}
$$

As in finite temperature, the result is independent of the ultraviolet cutoff. The above potential is monotonically decreasing (increasing) with $x$ if $q_{F}<q_{B} \leqslant 1 / 2 \quad\left(q_{B}<q_{F} \leqslant 1 / 2\right)$; therefore if only Eq. (3.3) is present, $\phi$ is driven to infinity (zero). We can expand Eq. (3.4) for $\phi \ll 1 / R \quad(x \ll 1)$ :

$$
\begin{aligned}
V(r, \phi)= & 3\left[\mathrm{Li}_{5}(r)+\mathrm{Li}_{5}\left(r^{*}\right)\right]-\frac{x^{2}}{2}\left[\mathrm{Li}_{3}(r)+\mathrm{Li}_{3}\left(r^{*}\right)\right] \\
& -\frac{x^{4}}{8} \ln \frac{(1-r)^{2}}{-r}+\frac{1}{15} x^{5}+\mathcal{O}\left(x^{6}\right) .
\end{aligned}
$$

This expansion is only valid if $r$ is not close to 1 , for $r$ $=1$, the expansion is

$$
\begin{aligned}
V(r= & 1, \phi)=6 \zeta(5)-\zeta(3) x^{2}+\left(\frac{3}{16}-\frac{1}{4} \log x\right) x^{4}+\frac{1}{15} x^{5} \\
& +\mathcal{O}\left(x^{6}\right),
\end{aligned}
$$

where $\zeta(x)$ is the Riemann-zeta function. Notice that the only odd-term in the $x$-power expansion of the potential $x^{5}$ cancels (see also Appendix B) in Eq. (3.3). This means that a cosmological phase transition in the $5 \mathrm{D}$ theory at temperatures $T>1 / R$, that can be described by means of a genuine 5D field theory at finite temperature, is always second order. A similar observation has been recently done in Ref. [17].

From Eqs. (3.3) and (3.6), we can obtain the mass of $\phi$ at the one-loop:

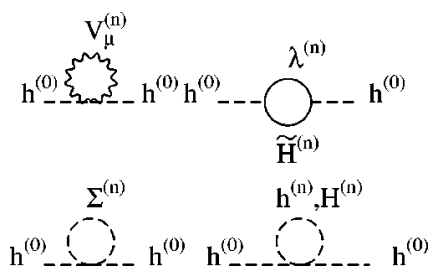

FIG. 1. Diagrams that contribute to the squared-mass of the Higgs boson field $h^{(0)}$.

$$
m_{\phi}^{2}=\left.\frac{1}{32 \pi^{4}} \operatorname{Tr}\left[\Delta m^{2}\left(q_{B}\right)-\Delta m^{2}\left(q_{F}\right)\right] \frac{d M^{2}(\phi)}{d|\phi|^{2}}\right|_{\phi=0},
$$

where

$$
\Delta m^{2}(q)=\frac{1}{2 R^{2}}\left[\mathrm{Li}_{3}\left(e^{i 2 \pi q}\right)+\mathrm{Li}_{3}\left(e^{-i 2 \pi q}\right)\right] .
$$

This coincides with the result in Ref. [32], and can be interpreted diagrammatically in terms of the diagrams of Fig. 1. In particular, we can calculate the one-loop mass of $h^{(0)}$ for the model described in the previous section in the case $q_{R}$ $=q_{H}=\omega$. Considering only the $\mathrm{SU}(2)_{L}$ interactions, Eq. (3.8) yields

$$
m_{\phi}^{2}=\frac{g_{2}^{2}}{64 \pi^{4}}\left[9 \Delta m^{2}(0)+3 \Delta m^{2}(2 \omega)-12 \Delta m^{2}(\omega)\right] .
$$

The Higgs boson squared mass at the origin, defined by Eq. (3.10), is positive definite and therefore radiative corrections on the boundary will be required to trigger EWSB, as we will see in the next sections. This procedure is a common one in theories where supersymmetry breaking is gauge mediated to the sector of squarks and sleptons. The value of $m_{\phi}$ defined by Eq. (3.10) is a monotonically increasing function of $\omega$ and takes values in the range $0<m_{\phi}<4 \times 10^{-2} / R$ for $0<\omega<1 / 2$. Thus the scalar remains around two orders of magnitude lighter than the compactification scale.

\section{B. Radiative corrections on the 4D boundary}

The scalar fields on the boundary (i.e., squarks and sleptons) are massless at tree level. Nevertheless, since supersymmetry is broken in the 5D bulk, the breaking will be transmitted to the fields on the boundary at the quantum level.

Let us consider the gauge corrections to the mass of $\widetilde{Q}$. The interactions between $\widetilde{Q}$ and the gauge supermultiplet are given in Eq. (2.8). At the one-loop level, the diagrams that contribute to $m_{\tilde{Q}}$ are given in Fig. 2. The first (last) two diagrams are provided by the interactions in the first (second) line of Eq. (2.8), while no contribution at one loop comes from the terms in the third line of Eq. (2.8).

We must sum over the full tower of KK excitations. Using the methods of Appendix B to sum over KK-states, the diagrams of Fig. 2 give 


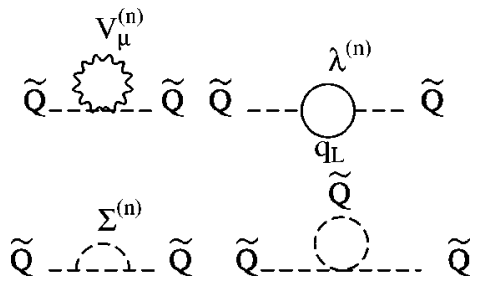

FIG. 2. Diagrams that contribute to the mass of $\widetilde{Q}$ from the gauge sector.

$$
m_{\widetilde{Q}}^{2}=\frac{g^{2} C_{2}(Q)}{4 \pi^{4}}\left[\Delta m^{2}(0)-\Delta m^{2}\left(q_{R}\right)\right]
$$

where $C_{2}(Q)$ is the quadratic Casimir of the $Q$-representation under the gauge group, ${ }^{7}$ and $\Delta m^{2}(q)$ is given in Eq. (3.9).

The interactions of $\widetilde{Q}$ with the Higgs boson sector can be read off from Eqs. (2.11) and (2.12). At the one-loop level the $h_{t}$ corrections to the mass of $\widetilde{Q}$ is provided by the diagrams of Fig. 3. The result is given by

$$
m_{\tilde{Q}}^{2}=\frac{h_{t}^{2}}{16 \pi^{4}}\left[\Delta m^{2}\left(q_{R}+q_{H}\right)+\Delta m^{2}\left(q_{R}-q_{H}\right)-2 \Delta m^{2}\left(q_{H}\right)\right] .
$$

A similar expression to Eq. (3.12) holds for the $h_{b}$ corrections.

In this way one can compute the radiative corrections to the masses of the sfermions $\widetilde{Q}, \widetilde{U}, \widetilde{D}, \widetilde{L}$, and $\widetilde{E}$, in the model presented in Sec. II. The result can be written as [32]

$$
\begin{aligned}
& m_{\widetilde{Q}}^{2}=\left(\frac{8}{6} \alpha_{3}+\frac{3}{4} \alpha_{2}+\frac{1}{60} \alpha_{1}\right) \Delta m_{g}^{2}+\frac{1}{2}\left(\alpha_{t}+\alpha_{b}\right) \Delta m_{H}^{2}, \\
& m_{\tilde{U}}^{2}=\left(\frac{8}{6} \alpha_{3}+\frac{4}{15} \alpha_{1}\right) \Delta m_{g}^{2}+\alpha_{t} \Delta m_{H}^{2} \\
& m_{\widetilde{D}}^{2}=\left(\frac{8}{6} \alpha_{3}+\frac{1}{15} \alpha_{1}\right) \Delta m_{g}^{2}+\alpha_{b} \Delta m_{H}^{2} \\
& m_{\tilde{L}}^{2}=\left(\frac{3}{4} \alpha_{2}+\frac{3}{20} \alpha_{1}\right) \Delta m_{g}^{2} \\
& m_{\tilde{E}}^{2}=\frac{3}{5} \alpha_{1} \Delta m_{g}^{2},
\end{aligned}
$$

where

\footnotetext{
${ }^{7} \mathrm{We}$ are using the convention for the generators $\operatorname{Tr} T_{R}^{\alpha} T_{R}^{\beta}$ $=T(R) \delta^{\alpha \beta}$ and $T_{R}^{\alpha} T_{R}^{\alpha}=C_{2}(R) \cdot \mathbf{1}$, where $R$ is a representation of the gauge group and the unit matrix 1 has dimension $d(R) \times d(R)$, where $d(R)$ is the dimensionality of $R$. In particular if $N$ is the fundamental representation of $\mathrm{SU}(N), T(N)=1 / 2$ and $C_{2}(N)$ $=\left(N^{2}-1\right) /(2 N)$, and for the adjoint (Adj) representation, $T($ Adj $)$ $=C_{2}(\operatorname{Adj})=N$.
}

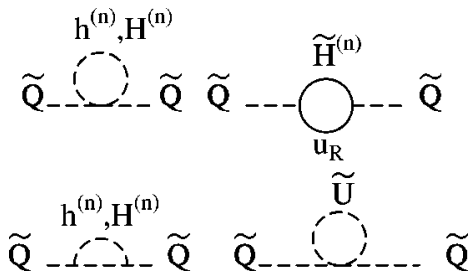

FIG. 3. Diagrams that contribute to the mass of $\widetilde{Q}$ from the Higgs boson sector.

$$
\Delta m_{g}^{2}=\left[\Delta m^{2}(0)-\Delta m^{2}\left(q_{R}\right)\right] / \pi^{3},
$$

and

$$
\Delta m_{H}^{2}=\left[\Delta m^{2}\left(q_{R}+q_{H}\right)+\Delta m^{2}\left(q_{R}-q_{H}\right)-2 \Delta m^{2}\left(q_{H}\right)\right] / 2 \pi^{3},
$$

with $\Delta m^{2}(q)$ given in Eq. (3.9). In Eq. (3.13) we have kept only the Yukawa couplings $h_{t, b}$ and defined $\alpha_{t, b}=h_{t, b}^{2} / 4 \pi$.

Finally we have computed the contribution of the KK towers to the soft-breaking trilinear couplings between two boundary and one bulk fields. This contribution arises from gaugino loops as depicted in the diagram of Fig. 4. The leading contribution to the parameter $A_{t}$ is provided by the exchange of gluinos and given by Ref. [32]:

$$
A_{t}=\frac{8}{6} \frac{\alpha_{3} h_{t}}{2 \pi^{2}}\left[i \mathrm{Li}_{2}\left(e^{i 2 \pi q_{R}}\right)-i \operatorname{Li}_{2}\left(e^{-i 2 \pi q_{R}}\right)\right] \frac{1}{R} \text {. }
$$

The mixing $A_{t}$ vanishes at $q_{R}=0,1 / 2$ and is bounded by $\left|A_{t}\right|<2 \times 10^{-2} / R$. The relative top squark mixing is rather small: the function $a_{t}\left(q_{R}\right) \equiv A_{t} / m_{\tilde{t}}$ is monotonically decreasing with $q_{R}$ and takes the values $0.33,0.18,0.12$ and zero for $q_{R}=1 / 10,1 / 4,1 / 3$, and $1 / 2$, respectively.

\section{ELECTROWEAK SYMMETRY BREAKING}

In Sec. II we have described the tree-level mass spectrum of all KK excitations. In particular we have seen that for $q_{R}=q_{H} \equiv \omega$ the zero mode $h^{(0)}$ is massless and thus a good candidate to become the SM Higgs boson. It is useful to know how $h^{(0)}$ is identified with the two Higgs bosons of the MSSM, $H_{1}$ and $H_{2}$. The latter are scalars with the SM quantum numbers $(\mathbf{1}, \mathbf{2}, 1 / 2)$ and $(\mathbf{1}, \mathbf{2},-1 / 2)$, that couple, respectively, to the down and up fermion sector. Therefore, we have from Eqs. (2.11) and (2.12)

$$
h^{(0)}=\frac{1}{\sqrt{2}}\left(\sigma^{2} H_{1}^{*}+H_{2}\right) \text {, }
$$

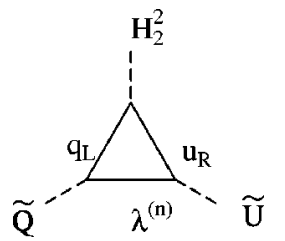

FIG. 4. Diagrams that contribute to $A_{t}$. 
TABLE III. Degrees of freedom and masses for KK modes.

\begin{tabular}{lcc}
\hline \hline Field & $d_{f}$ & Mass \\
\hline$V_{\mu}^{(n)}$ & $3 \times 3$ & $\frac{n^{2}}{R^{2}}+\frac{1}{4} g_{2}^{2} \phi^{2}$ \\
$\lambda^{(n)}$ & $3 \times 2$ & $\frac{(n+\omega)^{2}}{R^{2}}+\frac{1}{4} g_{2}^{2} \phi^{2}$ \\
$\widetilde{H}^{(n)}$ & $2 \times 2$ & $\frac{(n+\omega)^{2}}{R^{2}}+\frac{1}{2} C_{2}(H) g_{2}^{2} \phi^{2}$ \\
$H^{(n)}$ & 3 & $\frac{(n+2 \omega)^{2}}{R^{2}}+\frac{1}{4} g_{2}^{2} \phi^{2}$ \\
\hline \hline
\end{tabular}

where $\sigma^{2}$ acts on the $\mathrm{SU}(2)_{L}$ indices of the Higgs boson doublet. In the range $0<\omega<1 / 2$ all modes $H^{(n)}$ get masses proportional to $1 / R$ and become supermassive. In particular, the orthogonal field to Eq. (4.1), that includes the MSSM Higgs boson $H^{0}, H^{ \pm}$, and $A$, get a mass $2 \omega / R$, and then a zero VEV. This implies (if $h^{(0)}$ gets a nonzero VEV) that $\left\langle H_{2}\right\rangle=\left\langle H_{1}\right\rangle$ or $\tan \beta=1$ in the MSSM language. On the other hand, the Higgsino zero mode also gets a tree-level mass $\omega / R$ as we have seen in Table II. Therefore, there is no $\mu$ problem in this class of models.

In order to find out whether there is a nontrivial minimum that can induce EWSB, the effective potential of Eq. (3.3) has to be computed in the presence of the background field $\phi \equiv \sqrt{2}\left\langle h^{(0)}\right\rangle$. The effective potential in the bulk was given in Eq. (3.4) and the background dependent masses which appear in Eq. (3.5) can be read off from the 5D Lagrangian (2.1). The mass of all KK modes as well as the corresponding number of degrees of freedom $\left(d_{f}\right)$ coming from the SM group structure are displayed in Table III, where $g_{2}$ is the $\mathrm{SU}(2)_{L}$ gauge coupling. We are neglecting the $\mathrm{U}(1)_{Y}$ interactions. The counting of number of degrees of freedom is as follows. For the gauge fields we have three degrees of freedom coming from the $\mathrm{SU}(2)_{L}$ structure (a triplet) and three from the trace over Lorentz indices in the Landau gauge. For the gauginos there are three degrees of freedom from the $\mathrm{SU}(2)_{L}$ structure and two from the Majorana nature of gauginos. For the Higgsinos we have four degrees of freedom arising from their Dirac nature. Finally, in the Higgs boson sector, since there are no quartic couplings involving only the $h^{(n)}$ field, only some components of the $H^{(n)}$ field receive EWSB masses, in particular the charged components and the real part of the neutral component (the imaginary part does only receive the SS mass): a total of three degrees of freedom.

By using the squared-mass and degrees of freedom values from Table III in Eq. (3.3) we see that the squared-mass term in the potential $m_{\phi}^{2}$ is positive for all values of $\omega$ [see Eq. (3.10) and comments that follow it], which prevents the existence of a nontrivial EWSB minimum. However we have also to include in the one-loop potential the contribution from the 4D fields in the boundary. Technically speaking this is a two-loop contribution because the masses which appear

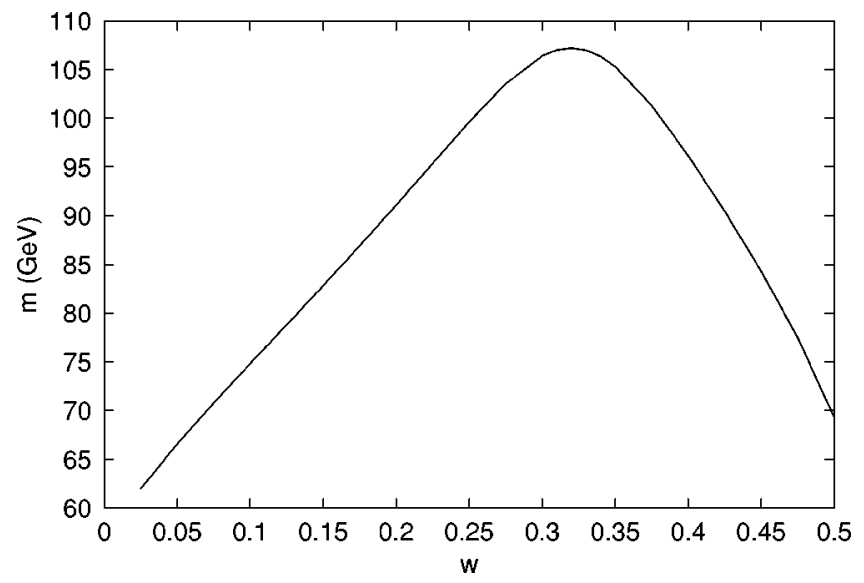

FIG. 5. Mass of the Higgs boson field coming from the minimization of the effective potential.

there were generated at one-loop, Eq. (3.13). However this correction can be numerically relevant, due to the smallness of the bulk-generated mass at the origin for the Higgs boson field, and must be the leading two-loop correction to the effective potential. This contribution takes the usual form

$$
V_{4 \mathrm{D}}=\frac{1}{64 \pi^{2}} \operatorname{Str} M^{4}\left(\log \frac{M^{2}}{\mathcal{Q}^{2}}-\frac{3}{2}\right),
$$

where $M$ is the mass of the particle on the boundary which includes the EWSB contribution from the Higgs boson field $h^{(0)}$ and $\mathcal{Q}$ is a renormalization scale. The only relevant contribution to Eq. (4.2) is that coming from the top/stop sector. ${ }^{8}$ Now the one-loop potential (4.2) is similar to the MSSM one, once we have introduced the soft-breaking parameters from Eqs. (3.13) and (3.16). Finally we will choose the renormalization scale $\mathcal{Q}$ as the boundary fixed by the lightest tree-level mass below which the theory can be considered $4 \mathrm{D}$, i.e., the gaugino or Higgsino mass $\omega / R$.

Now we proceed in the following way. For a fixed value of $0<\omega<1 / 2$ we write the full effective potential, Eqs. (3.3) plus (4.2), as a function of $\phi$ and $R$. By imposing the condition that $\langle\phi\rangle=246 \mathrm{GeV}$ we fix the value of the fifth dimension radius ${ }^{9} R$ as a function of $\omega$ and we can deduce from it the value of all soft-supersymmetry breaking parameters as well as the mass of the light physical Higgs boson $h^{(0)}$. The latter is shown in Fig. 5 as a function of $\omega$. We see from Fig. 5 that the maximum value is achieved when $\omega$ $\sim 1 / 3$, and that for this particular value of $\omega$ the Higgs boson mass is $\sim 107 \mathrm{GeV}$. The presence of a light Higgs boson is due to the fact that $h^{(0)}$ is a flat direction of the $D$-term potential [30] and therefore its quartic coupling is zero at tree-level. The spectrum for $\omega=\frac{1}{3}$ is presented in Table IV

\footnotetext{
${ }^{8}$ We can neglect the sbottom sector, since for $\tan \beta=1$, this would provide subdominant contributions.

${ }^{9} \mathrm{We}$ are not considering the compactification radius as a dynamical variable. Some ideas on how to dynamically fix the value of $R$ can be found in Ref. [32].
} 
TABLE IV. Supersymmetric chiral spectrum for the model with $\omega=\frac{1}{3}$.

\begin{tabular}{lcccc}
\hline \hline $1 / R$ & $m_{\lambda}$ & $m_{\tilde{Q}}$ & $m_{\tilde{L}}$ & $m_{\tilde{E}}$ \\
\hline $24 \mathrm{TeV}$ & $8 \mathrm{TeV}$ & $2.2 \mathrm{TeV}$ & $1 \mathrm{TeV}$ & $496 \mathrm{GeV}$ \\
\hline \hline
\end{tabular}

for the supersymmetric chiral matter, and in Table $\mathrm{V}$ for the Higgs boson sector. For this case the shape of the effective potential is shown in Fig. 6.

We also plot in Fig. 7 the mass spectrum of this model as a function of $\omega$. It is worth noting that the overall shape is similar in every case and also similar to the one in Fig. 5.

As can be seen in Tables IV, V this model predicts a rather heavy spectrum of supersymmetric particles. In spite of this fact (and unlike the case of the MSSM with a similar mass spectrum) the mass gap between the electroweak scale $[\mathcal{O}(100) \mathrm{GeV}]$ and the supersymmetric spectrum $[\mathcal{O}($ few $) \mathrm{TeV}]$ is generated by finite radiative corrections (without any large logarithm as in the MSSM) and there is no fine-tuning in the usual sense. Notice that the only independent parameter in our theory is $\omega$ and therefore there is no possibility to tune the spectrum. The heavy spectrum of Fig. 7 is in fact an unavoidable prediction of the theory. Another characteristic feature of the spectrum is that the LSP is the right-handed selectron, due to the fact that supersymmetry breaking is transmitted via gauge interactions. Having the right-handed selectron as the LSP presents some cosmological difficulties. A possible way out is that $R$ parity is violated, so as the LSP is not stable. Other possibilities were discussed in Ref. [32]. We must remark that the results on the light Higgs boson mass are quite sensitive to the value of $\mathcal{Q}$ in Eq. (4.2); a little variation in this value, turns out to make a large variation in its mass. This implies that there is a certain uncertainty in the actual value of $m_{h}$, that would be aminorated if the two-loop contributions are incorporated. Nevertheless, we think that this two-loop calculation is not needed yet, since the experimental uncertainties on the values of $h_{t}$ and $\alpha_{3}$ are still large. Furthermore we do not expect that the overall shape of the spectrum would be changed by these two-loop corrections in the bulk.

Let us finally consider the case $\omega=1 / 2$. As we said, we have in this case two massless scalars in the spectrum, $h^{(0)}$ and $H^{(-1)}$, that can be associated with the two Higgs bosons of the MSSM:

$$
\begin{aligned}
h^{(0)} & =\frac{1}{\sqrt{2}}\left[\sigma^{2} H_{1}^{*}+H_{2}\right], \\
H^{(-1)} & =\frac{1}{\sqrt{2}}\left[\sigma^{2} H_{1}^{*}-H_{2}\right] .
\end{aligned}
$$

TABLE V. Higgs boson sector spectrum for the model with $\omega$ $=\frac{1}{3}$.

\begin{tabular}{lcccc}
\hline \hline $\tan \beta$ & $m_{\tilde{H}}$ & $m_{H^{ \pm}}=m_{H^{0}}$ & $m_{A}$ & $m_{h}$ \\
\hline 1 & $8 \mathrm{TeV}$ & $15.9 \mathrm{TeV}$ & $15.8 \mathrm{TeV}$ & $107 \mathrm{GeV}$ \\
\hline \hline
\end{tabular}

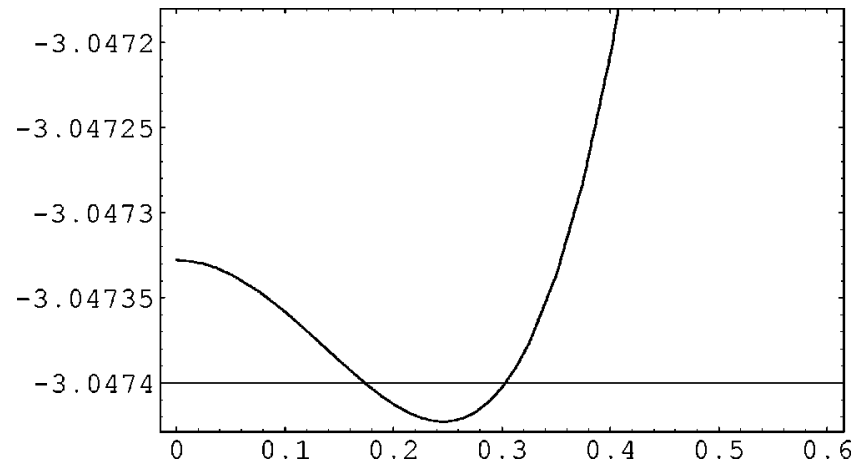

FIG. 6. Effective potential (in $\mathrm{TeV}^{4}$ ) as a function of $\phi$ (in $\mathrm{TeV})$.

Nevertheless, this model is phenomenologically problematic since in the limit $\omega=1 / 2$ the theory has a residual symmetry that does not allow for an $H_{1} H_{2}$ mixing term (i.e., $B \mu=0$ in the MSSM language) and therefore the VEV of $H_{1}$ is zero. To see that notice that for $\omega=1 / 2$ the Higgsino $n$ KK mode (in Table II) is degenerated with the $-n-1 \mathrm{KK}$ mode, and the $h^{(n)} \mathrm{KK}$ mode with the $H^{(-n-1)}$. Therefore the theory in the bulk and the boundary is invariant under the discrete transformation

$$
\begin{aligned}
& h^{(n)} \leftrightarrow H^{(-n-1)}, \\
& \widetilde{H}_{L}^{(n)} \leftrightarrow-\widetilde{H}_{L}^{(-n-1)}, \\
& \widetilde{H}_{R}^{(n)} \leftrightarrow \widetilde{H}_{R}^{(-n-1)}, \\
&\left(\widetilde{Q}, q_{L}\right) \leftrightarrow\left(\widetilde{Q}, q_{L}\right), \\
&\left(\widetilde{U}, u_{R}\right) \leftrightarrow-\left(\widetilde{U}, u_{R}\right), \\
&\left(\widetilde{D}, d_{R}\right) \leftrightarrow\left(\widetilde{D}, d_{R}\right) .
\end{aligned}
$$

This symmetry implies $H_{1} \rightarrow H_{1}$ and $H_{2} \rightarrow-H_{2}$ and consequently no mixing-mass term between the MSSM Higgses

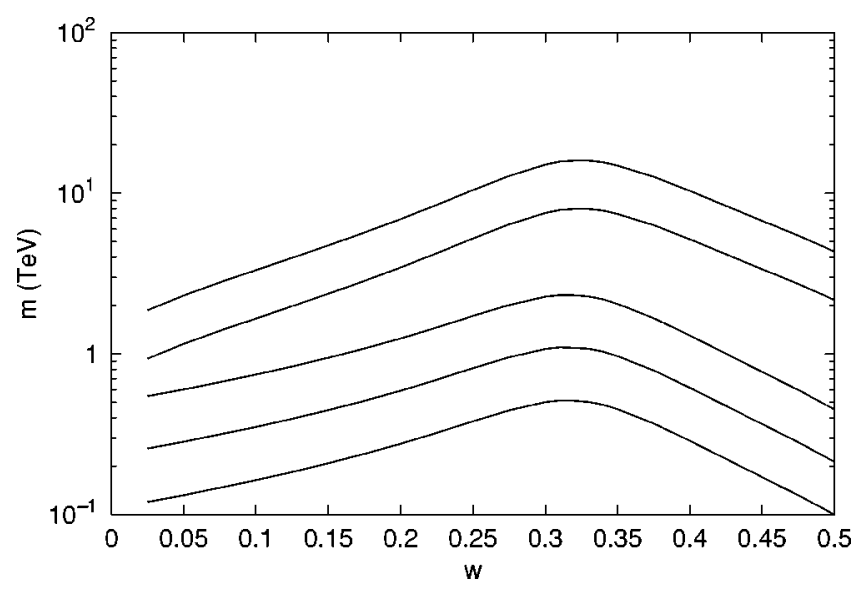

FIG. 7. Masses of the different supersymmetric particles of the spectrum. From top to bottom we have the Higgs boson doublet, the gauginos and Higgsinos, the squarks, the left-handed sleptons, and the right-handed sleptons. 
can be generated at any loop order. Note also that in the limit $\omega=1 / 2$ the theory has also an $R$ symmetry, since the two Majorana gauginos $\lambda^{(n)}$ and $\lambda^{(-n-1)}$ can combine to form a Dirac fermion. Consequently the trilinear terms (3.16) are not generated in the limit $\omega=1 / 2$.

\section{AN ALTERNATIVE MODEL}

In this section we want to present a different possibility from the model studied above. We will assume quarks and leptons live in the bulk but the two Higgs boson supermultiplets live on the boundary. This possibility, although it suffers from the $\mu$ problem, presents a different and interesting phenomenology.

Quarks and leptons can arise from the zero modes of hypermultiplets. Compactifying in $S^{1} / Z_{2}$, we can obtain a chiral theory with $N=1$ supersymmetry. ${ }^{10}$ We can again use the SS mechanism to break supersymmetry. Taking $q_{R} \neq 0$, the squarks and sleptons get masses at the tree-level equal to $q_{R} / R$, and the massless sector in the bulk corresponds to the SM fermions and (as in the model above) to the SM gauge bosons. Now, however, since the two Higgs boson doublets live on the boundary, the scalar Higgs bosons and Higgsinos are massless at tree-level. As in the model above, the scalar Higgs bosons will get masses from their interactions with the bulk $^{11}$ but the Higgsinos will remain massless at any loop order; the model suffers from the $\mu$ problem. To make the model phenomenological viable a Higgsino mass must be generated. A simple way to give mass to the Higgsino is through a nonzero VEV of a singlet $S$ field coupled to $H_{1} H_{2}$. We will not specify here how $S$ gets a VEV. We just want to point out that the phenomenology of this scenario is quite different from the previous one. The Higgsinos are the LSPs. Since the gaugino mass is very large, the charged Higgsino will be slightly heavier than the neutral Higgsino, with a mass difference of few GeVs [36]. Therefore the LSP is a neutral particle that does not present the cosmological problems of a charged one. The mass degeneracy of the Higgsinos, however, makes their detection problematic. The usual decay channel $\widetilde{H}^{+} \rightarrow \widetilde{H}^{0} e \nu$ cannot be used to detect $\widetilde{H}^{+}$, because of the lack of energy of the $e \nu$ decay products. In this case, the detection of the charged Higgsino must be carried out by photon tagging.

\footnotetext{
${ }^{10}$ In a string theory, if the Higgs bosons live on the boundary (twisted sector of the orbifold) and the quarks live in the bulk (untwisted sector), the Yukawa couplings can only be generated (to respect the $Z_{2}$ symmetry of the orbifold) through nonrenormalizable couplings, as, e.g., $\mathrm{XH}_{2} q_{L} u_{R}$, where $X$ is a SM singlet living on the boundary (twisted sector) which must acquire a VEV of the order of the high-scale $\Lambda$ (cutoff)

${ }^{11}$ Notice that in this case, the finite one-loop contribution to the mass of $\mathrm{H}_{2}$ arising from the top quark or top squark sector (that live in the bulk) can be negative [this is given by Eq. (3.12) with $q_{H}$ $=0]$ and dominate over the positive gauge contribution [Eq. (3.11)]. This would make the EWSB easier and lead to a heavier lightest Higgs boson mass.
}

\section{UNIFICATION WITH SS SUPERSYMMETRY BREAKING}

The phenomenology of gauge coupling unification in the presence of extra dimensions was studied in Refs. [9] where it was proven that a sufficient condition for unification is that the ratio

$$
R_{i j}=\frac{b_{i}^{\mathrm{KK}}-b_{j}^{\mathrm{KK}}}{b_{i}^{\mathrm{MSSM}}-b_{j}^{\mathrm{MSSM}}},
$$

does not depend on $(i, j)$, where $b_{i}^{\text {MSSM }}$ are the MSSM $\beta$-function coefficients and $b_{i}^{\mathrm{KK}}$ those of the $N=2 \mathrm{KK}$ excitations. Using $b^{\mathrm{MSSM}}=(33 / 5,1,-3)$ and $b^{\mathrm{KK}}=(6 / 5,-2$, -6) for the model presented in Sec. II and in Ref. [30], we can see that it does not satisfy the necessary requirements to fulfill gauge coupling unification. This fact has recently motivated the suggestion of enlarging the model with the extra hypermultiplets $\mathbb{F}^{a}(a=1,2) \quad[8]$ which are $\mathrm{SU}(3)_{c}$ $\times \mathrm{SU}(2)_{L}$ singlets and having hypercharge $Y=1$. In Ref. [8] it was proven that the enlarged model unifies as well as the MSSM provided that we can introduce a supersymmetric mass term $\mu_{F} \leq 1 / R$ for the new fields, that can be done by means of singlet fields getting nonzero VEVs. In this section we will discuss how to incorporate the fields $\mathbb{F}^{a}$ in our formalism where the SS mechanism breaks supersymmetry and how the extra $\mu$ problem can be solved in a similar fashion as the $\mu$ problem for Higgs boson fields. Also we will study the issue of gauge coupling unification in the presence of SS supersymmetry breaking.

Introduction of the hypermultiplets $\mathbb{F}^{a}$ in our formalism can be done along the same lines as those leading to the mass spectrum and interactions of the hypermultiplets $\mathrm{H}^{a}$ in Sec. II A. In fact, the 5D Lagrangian for the vector multiplet (V) and the hypermultiplets $\mathbb{F}^{a}$ is given by Eq. (2.1) after replacing $\mathbb{H}^{a} \rightarrow \mathbb{F}^{a}$, yielding mass eigenvalues as those given in Table II after the replacement $q_{H} \rightarrow q_{F}$. There is also the coupling of matter supermultiplets on the boundary with the hypermultiplets $\mathbb{F}^{a}$ corresponding to the last term of Eqs. (2.7) and (2.8). Note that by choosing $q_{F} \neq q_{R}$ no massless modes do appear in the spectrum and all zero modes will acquire masses $\leqslant 1 / R$, as required by gauge coupling unification, without any need to introduce supersymmetric mass terms.

For scales $\mathcal{Q} \ll 1 / R$ the standard Coleman-Weinberg prescription for the gauge couplings gives the one-loop result for the previous model,

$$
\begin{aligned}
\alpha_{i}^{-1}(\mathcal{Q})= & \alpha_{i}^{-1}(\Lambda)+\frac{b_{i}^{\mathrm{SM}}}{2 \pi} \ln \frac{M_{c}}{\mathcal{Q}}+\frac{b_{i}^{\mathrm{MSSM}}-b_{i}^{\mathrm{KK}}}{2 \pi} \ln \frac{\Lambda}{M_{c}} \\
& +\frac{1}{2} \frac{1}{4 \pi} \sum_{f \in \mathrm{V}, \mathrm{H}^{a}, \mathbb{F}^{a}} b^{(f)} T\left(R_{f}\right) \\
& \times \sum_{n=-\infty}^{\infty} \int_{r \Lambda^{-2}}^{r M_{c}^{-2}} \frac{d t}{t} e^{-\left(n+\omega_{f}\right)^{2} t M_{c}^{2}},
\end{aligned}
$$


where the mass of the $n$th KK excitation of the $f$ field is ( $n$ $\left.+\omega_{f}\right) / R$, we have already introduced the $\mathbb{Z}_{2}$ projection, $M_{c}$ $=1 / R$, the cutoff coefficient is [9] $r=\pi / 4, b^{\mathrm{SM}}=(41 / 10$, $-19 / 6,-7)$ and the $\beta$-function coefficients $b^{(f)}$ are $b($ gauge boson $)=-11 / 3, \quad b($ Weyl fermion $)=2 / 3, \quad$ and $b($ complex scalar $)=1 / 3$. They obviously satisfy the condition $\Sigma_{f} b^{(f)} T\left(R_{f}\right)=b_{i}^{\mathrm{KK}}$ where, for the enlarged MSSM model,

$$
b^{\mathrm{KK}}=(18 / 5,-2,-6) \text {. }
$$

The last integral in Eq. (6.2)

$$
I(\omega)=\sum_{n=-\infty}^{\infty} \int_{r\left(M_{c} / \Lambda\right)^{2}}^{r} \frac{d x}{x} e^{-(n+\omega)^{2} x},
$$

can be computed with the help of the Poisson resummation formula

$$
\sum_{n=-\infty}^{\infty} e^{-(n+\omega)^{2} x}=\sqrt{\frac{\pi}{x}} \sum_{n=-\infty}^{\infty} e^{-\left(\pi^{2} / x\right) n^{2}-2 i \pi n \omega},
$$

and it can be approximated by

$$
\begin{aligned}
I(\omega) & =4\left(\frac{\Lambda}{M_{c}}-1+\frac{1}{2} \sum_{n=1}^{\infty} \frac{\cos (2 \pi n \omega)}{n}[1-\operatorname{Erf}(2 n \sqrt{\pi})]\right) \\
& \simeq 4\left(\frac{\Lambda}{M_{c}}-1+\frac{1}{4 \pi} e^{-4 \pi} \cos (2 \pi \omega)\right)
\end{aligned}
$$

where Erf is the error function and we have used its asymptotic expansion, which is dominated by the $n=1$ mode. The function (6.6) exhibits a tiny $\omega$ dependence since $e^{-4 \pi} / 4 \pi \sim 10^{-7}$. Therefore Eq. (6.2) looks similar to

$$
\begin{aligned}
\alpha_{i}^{-1}(\mathcal{Q})= & \alpha_{i}^{-1}(\Lambda)+\frac{b_{i}^{\mathrm{SM}}}{2 \pi} \ln \frac{M_{c}}{\mathcal{Q}}+\frac{b_{i}^{\mathrm{MSSM}}-b_{i}^{\mathrm{KK}}}{2 \pi} \ln \frac{\Lambda}{M_{c}} \\
& +\frac{b_{i}^{\mathrm{KK}}}{2 \pi}\left(\frac{\Lambda}{M_{c}}-1\right),
\end{aligned}
$$

and unification proceeds, concerning KK-modes, as in the supersymmetric case.

\section{CONCLUSIONS}

In this paper we have addressed the issue of extra dimensions at the $\mathrm{TeV}$ scale as a possible origin of electroweak breaking for the standard model, as well as the source of soft breaking terms in its supersymmetric extensions. In a bottom-up approach we have constructed a minimal extension of the MSSM in a five-dimensional space-time, with one "large", space dimension with radius $\sim 1 \mathrm{TeV}^{-1}$, which exhibits all the required features.

After compactification on the segment $S^{1} / Z_{2}$ the zero modes of the model constitute the spectrum of the MSSM, while the nonzero Kaluza-Klein excitations are arranged into $N=2$ supermultiplets. When supersymmetry is broken by the Scherk-Schwarz mechanism, making use of the underly- ing $N=2 \mathrm{SU}(2)_{R}$ algebra, the massless spectrum in the Higgs boson and gauge sector coincides with that of the pure standard model while their fermionic partners acquire treelevel masses. Chiral sfermions are massless at the tree-level, since chiral matter is supposed to live on the four dimensional boundary of the fifth dimension.

Supersymmetry is gauge and Yukawa mediated to the chiral sector by radiative corrections, which also trigger electroweak symmetry breaking. In those aspects the model shares common features with any gauge-mediated supersymmetry breaking model but with a very characteristic spectrum. Electroweak breaking is achieved with a rather light SM Higgs boson (lighter than $\sim 110 \mathrm{GeV}$ ), very heavy gauginos, Higgsinos and non-SM Higgs bosons (with masses $\sim 1 / R)$, and chiral sfermions at some intermediate squared masses $\left(\sim \alpha_{i} / R^{2}\right)$ where $\alpha_{i}$ are either gauge or Yukawa couplings. The model does not suffer from any $\mu$ problem since there is an effective $\mu$ parameter $\sim 1 / R$ : in fact both the Higgsinos and the pseudoscalar Higgs boson acquire masses $\sim 1 / R$.

Gauge coupling running and unification in the presence of extra dimensions is studied when supersymmetry is broken by the Scherk-Schwarz mechanism. Concerning the running, in the presence of the fifth dimension, we have computed the contribution from a tower of KK-excitations with a mass given by $(n+\omega) / R$. The leading contribution, $\sim(\Lambda R-1)$, is $\omega$ independent, while all the $\omega$ dependence is concentrated in subleading contributions which are corrections $\sim 10^{-7} \cos (2 \pi \omega)$, and therefore negligible.

The model we have presented in this paper must not be considered as a unique model, with unique predictions, but rather as a representative of a class of models sharing common features: there is some extra dimension(s) at the $\mathrm{TeV}$ scale which triggers, through the Scherk-Schwarz mechanism, the breaking of supersymmetry and the electroweak symmetry. Other possibilities, apart from the minimal model we have studied in great detail, have been pointed out, that offer a wide and rich variety of different phenomenological outcomes. Since the common feature of all these models is the appearance of extra dimensions at the $\mathrm{TeV}$ scale, they are testable at present and future accelerators, which makes it worth pursuing their analysis and, in particular, the search of their experimental signatures. From a more fundamental point of view it would be important to find consistent string vacua reducing to our models at low energies: e.g., D4 branes in compactifications of type-IIA orientifolds or D5branes in type-I $\left(-\mathrm{I}^{\prime}\right)$ or type-IIB orientifolds. Some of these ideas are at present being investigated $[6,8,37]$.

\section{ACKNOWLEDGMENTS}

We thank Ignatios Antoniadis, Karim Benakli, Savas Dimopoulos and Zurab Kakushadze for discussions. The work of AD was supported by the Spanish Education Office (MEC). This work was supported in part by the CICYT of Spain (contracts AEN98-0816 and AEN95-0882). 


\section{APPENDIX A: GAUGE FIXING}

In this appendix we will show that, in the 5D Landau gauge, the Goldstone boson $V_{5}^{(n)}$ decouples from the Lagrangian. This is a nice feature of the five-dimensional theory.

We start with the kinetic gauge boson Lagrangian plus a gauge fixing term ${ }^{12}$ in five dimensions:

$$
\mathcal{L}_{5}=-\frac{1}{4} F^{M N} F_{M N}-\frac{1}{2 \xi}\left(\partial_{M} V^{M}\right)^{2} .
$$

Dimensional reduction of Eq. (A1) leads to the following expression:

$$
\begin{aligned}
\mathcal{L}_{4}= & \sum_{n=0}^{\infty}\left(-\frac{1}{4} F^{(n) \mu \nu} F_{\mu \nu}^{(n)}+\frac{n^{2}}{2 R^{2}} V_{\mu}^{(n)} V^{(n) \mu}\right. \\
& \left.-\frac{1}{2 \xi}\left(\partial_{\mu} V^{(n) \mu}\right)^{2}\right)+\sum_{n=1}^{\infty}\left[\frac{1}{2} \partial_{\mu} V_{5}^{(n)} \partial^{\mu} V_{5}^{(n)}-\frac{n^{2}}{2 \xi R^{2}} V_{5}^{(n) 2}\right. \\
& \left.+\frac{n}{R}\left(\frac{1}{\xi}-1\right) \partial_{\mu} V^{(n) \mu} V_{5}^{(n)}\right] .
\end{aligned}
$$

The propagators for $V_{\mu}^{(n)}$ and $V_{5}^{(n)}$, using Eq. (A2), are given by

$$
\begin{aligned}
\left\langle V_{\mu}^{(n)} V_{\nu}^{(n)}\right\rangle & =\frac{-i}{p^{2}-n^{2} / R^{2}}\left(\eta_{\mu \nu}+(\xi-1) \frac{p_{\mu} p_{\nu}}{p^{2}-\xi\left(n^{2} / R^{2}\right)}\right), \\
\left\langle V_{5}^{(n)} V_{5}^{(n)}\right\rangle & =\frac{i}{p^{2}-n^{2} / \xi R^{2}} .
\end{aligned}
$$

Taking now the limit $\xi \rightarrow 0$ in Eq. (A3) we obtain the normal propagator for a massive gauge boson in the Landau gauge, but we note that the propagator for the Goldstone boson vanishes, so $V_{5}^{(n)}$ decouples from the Lagrangian for each mode. The conclusion is that the Landau gauge in five dimensions leads to the Landau gauge in four dimensions for the gauge bosons and to the unitary gauge for the Goldstone bosons.

\section{APPENDIX B: THE EFFECTIVE POTENTIAL}

In this appendix we will compute the effective potential corresponding to a tower of bosonic and fermionic $\mathrm{KK}$ modes with masses given by Eq. (3.1). The basic integral we have to compute is then

$$
V=\frac{1}{2} \int \frac{d^{4} p}{(2 \pi)^{4}} \sum_{n=-\infty}^{\infty} \ln \left[l^{2} E^{2}+(n+\omega)^{2} \pi^{2}\right]
$$

\footnotetext{
${ }^{12} \mathrm{We}$ consider first an Abelian theory, the translation to the nonAbelian case being straightforward.
}

where $l=\pi R$ is the length of the segment, $\omega$ is either $q_{B}$ or $q_{F}$ in Eq. (3.2), a global minus sign has to be added in the case of fermions, and $E^{2}=p^{2}+M^{2}(\phi)$.

We will first evaluate the infinite sum over the KK modes in

$$
W=\frac{1}{2} \sum_{n=-\infty}^{\infty} \ln \left[(l E)^{2}+(n+\omega)^{2} \pi^{2}\right]
$$

or equivalently, in

$$
\frac{\partial W}{\partial E}=l^{2} E \sum_{n=-\infty}^{\infty} \frac{1}{(l E)^{2}+(n+\omega)^{2} \pi^{2}},
$$

by means of well known techniques used in field theory at finite temperature. To this end we will make use of the identity

$$
\frac{1}{(l E)^{2}+(n+\omega)^{2} \pi^{2}}=2 i \lim _{z \rightarrow(n+\omega) \pi} \frac{z-(n+\omega) \pi}{e^{2 i z}-e^{2 i \omega \pi}} \frac{e^{2 i \omega \pi}}{(l E)^{2}+z^{2}},
$$

and replace the infinite sum in Eq. (B3) by an integral in the complex plane $z$ over a contour which is the sum of the contours encircling anticlockwise the infinite number of poles along the real axis at $z=(n+\omega) \pi$. We now deform this contour into a contour going from $+\infty+i \epsilon$ to $-\infty+i \epsilon$ (which can be closed clockwise at $|z| \rightarrow \infty$ ) and a contour going from $-\infty-i \epsilon$ to $+\infty-i \epsilon$ (which can equally be closed clockwise at $|z| \rightarrow \infty)$, and make use of the residues theorem to perform the integral over $z$. This easily yields

$$
W=l E+\frac{1}{2}\left\{\ln \left(1-r e^{-2 l E}\right)+\ln \left(1-\frac{1}{r} e^{-2 l E}\right)\right\},
$$

where $r=\exp (-2 i \omega \pi)$, which corresponds to the decomposition of the effective potential as

$$
V=V^{(\infty)}+V^{(R)}
$$

The first term in Eq. (B6) comes from the first term in Eq. (B5) and provides a genuine $5 \mathrm{D}$ effective potential. It corresponds to the decompactification limit $(R \rightarrow \infty)$ of the theory and it is similar to the zero-temperature term which is obtained in field theory at finite temperature calculations. Since the $5 \mathrm{D}$ theory is not renormalizable, it must be computed by introducing a physical cutoff $\Lambda$ in the integral. Then the integral can be given an analytical form as

$$
\begin{aligned}
V^{(\infty)} & =l \int \frac{d^{4} p}{\left(2 \pi^{4}\right)} \sqrt{p^{2}+M^{2}(\phi)} \\
& =\frac{l}{16 \pi^{2}}\left\{\frac{4}{15} M^{5}+\frac{2}{15} \sqrt{\Lambda^{2}+M^{2}}\left(3 \Lambda^{4}+\Lambda^{2} M^{2}-2 M^{4}\right)\right\} .
\end{aligned}
$$

Notice that upon expansion of Eq. (B7) in powers of $M$ the only odd power is given by the $M^{5}$ term. This term cancels 
the similar one in the expansion of $V^{(R)}$ [see Eq. (3.6)] and there is no odd-power term in the expansion of the effective potential. ${ }^{13}$ However, in a supersymmetric theory $V_{B}^{(\infty)}$ $=V_{F}^{(\infty)}$ and the contributions to the effective potential from

\footnotetext{
${ }^{13}$ This is in contradistinction with the case of a 4D theory at finite temperature where there is an $M^{3}$ term in the bosonic expansion, which triggers first-order phase transitions. As a consequence, in a 5D theory at finite temperature the phase transition should be of second order.
}

$V^{(\infty)}$ do cancel. The second term in Eq. (B6) comes from the last two terms in Eq. (B5) which yields, upon integration over angular variables,

$$
V^{(R)}=\frac{1}{32 \pi^{6} R^{4}} \int_{0}^{\infty} d y y\left[\ln \left(1-r e^{-2 \sqrt{y+(\pi R M)^{2}}}\right)+\left(r \rightarrow \frac{1}{r}\right)\right] .
$$

Finally, the $y$ integral can be performed analytically giving the result that can be found in Eqs. (3.3) and (3.4).
[1] I. Antoniadis, Phys. Lett. B 246, 377 (1990); Proceedings of the PASCOS-91 Symposium, Boston, 1991 (World Scientific, Singapore, 1991), p. 718.

[2] T. Banks and M. Dine, Nucl. Phys. B479, 173 (1996); B505, 445 (1997).

[3] E. Cáceres, V. Kaplunovsky, and I.M. Mandelberg, Nucl. Phys. B493, 73 (1997).

[4] I. Antoniadis and M. Quirós, Phys. Lett. B 392, 61 (1997).

[5] E. Witten, Nucl. Phys. B471, 135 (1996); P. Horava and E. Witten, ibid. B460, 506 (1996); B475, 94 (1996).

[6] J. Lykken, Phys. Rev. D 54, 3693 (1996); G. Shiu and S.-H.H. Tye, ibid. 58, 106007 (1998); Z. Kakushadze and S.-H. Tye, ibid. 58, 126001 (1998); Nucl. Phys. B548, 180 (1999).

[7] I. Antoniadis, N. Arkani-Hamed, S. Dimopoulos, and G. Dvali, Phys. Lett. B 436, 257 (1998).

[8] Z. Kakushadze, Nucl. Phys. B548, 205 (1999); B552, 3 (1999).

[9] K.R. Dienes, E. Dudas, and T. Gherghetta, Phys. Lett. B 436, 55 (1998); 537, 47 (1999); “TeV-scale GUTs,', hep-ph/9807522.

[10] C. Bachas, J. High Energy Phys. 11, 023 (1998).

[11] D. Ghilencea and G.G. Ross, Phys. Lett. B 442, 165 (1998).

[12] T. Kobayashi, J. Kubo, M. Mondragon, and G. Zoupanos, Nucl. Phys. B550, 99 (1999).

[13] N. Arkani-Hamed, S. Dimopoulos, G. Dvali, and J. MarchRussell, "Neutrino Masses from Large Extra Dimensions," hep-ph/9811448; K.R. Dienes, E. Dudas, and T. Gherghetta, "Neutrino Oscillations without Neutrino Masses or Heavy Mass Scales: A Higher-Dimensional Seesaw Mechanism,', hep-ph/9811428.

[14] N. Arkani-Hamed, S. Dimopoulos, and G. Dvali, Phys. Lett. B 429, 263 (1998); N. Arkani-Hamed, S. Dimopoulos, and J. March-Russell, Phys. Rev D (to be published), hep-th/ 9809124.

[15] H. Hatanaka, T. Inami, and C.S. Lim, Mod. Phys. Lett. A 13, 2601 (1998).

[16] R. Sundrum, Phys. Rev. D 59, 085009 (1999); 59, 085010 (1999).

[17] K.R. Dienes, E. Dudas, T. Gherghetta, and A. Riotto, Nucl. Phys. B543, 387 (1999).

[18] I. Antoniadis and C. Bachas, Phys. Lett. B 450, 83 (1999).

[19] E.A. Mirabelli and M. Peskin, Phys. Rev. D 58, 065002 (1998).

[20] I. Antoniadis, S. Dimopoulos, and G. Dvali, Nucl. Phys. B516, 70 (1998).
[21] N. Arkani-Hamed, S. Dimopoulos, and G. Dvali, Phys. Rev. D 59, 086004 (1999); K. Benakli, ibid. (to be published), hep-ph/ 9809582; S. Nussinov and R. Shrock, ibid. 59, 105002 (1999); Z. Berezhiani and G. Dvali, Phys. Lett. B 450, 24 (1999).

[22] I. Antoniadis, K. Benakli, and M. Quirós, Phys. Lett. B 331, 313 (1994)

[23] G.F. Giudice, R. Rattazzi, and J.D. Wells, Nucl. Phys. B544, 3 (1999); E.A. Mirabelli, M. Perelstein, and M.E. Peskin, Phys. Rev. Lett. 82, 2236 (1999); T. Han, J.D. Lykken, and R.-J. Zhang, Phys. Rev. D 59, 105006 (1999); J.L. Hewett, Phys. Rev. Lett. 82, 4765 (1999); P. Mathews, S. Raychaudhuri, and K. Sridhar, Phys. Lett. B 450, 343 (1999).

[24] J. Scherk and J.H. Schwarz, Nucl. Phys. B153, 61 (1979); Phys. Lett. 82B, 60 (1979); E. Cremmer, J. Scherk, and J.H. Schwarz, ibid. 84B, 83 (1979); P. Fayet, Phys. Lett. 159B, 121 (1985); Nucl. Phys. B263, 649 (1986).

[25] R. Rohm, Nucl. Phys. B237, 553 (1984); C. Kounnas and M. Porrati, ibid. B310, 355 (1988); S. Ferrara, C. Kounnas, M. Porrati, and F. Zwirner, ibid. B318, 75 (1989); C. Kounnas and B. Rostand, ibid. B341, 641 (1990); E. Kiritsis, C. Kounnas, P.M. Petropoulos, and J. Rizos, Phys. Lett. B 385, 87 (1996).

[26] G. Dvali and M. Shifman, Nucl. Phys. B504, 127 (1997).

[27] I. Antoniadis and M. Quirós, Nucl. Phys. B505, 109 (1997); Phys. Lett. B 416, 327 (1998); Nucl. Phys. B (Proc. Suppl.) 62A-C, 312 (1998).

[28] E. Dudas and C. Grojean, Nucl. Phys. B507, 553 (1997); E. Dudas, Phys. Lett. B 416, 309 (1998).

[29] I. Antoniadis, C. Muñoz, and M. Quirós, Nucl. Phys. B397, 515 (1993).

[30] A. Pomarol and M. Quirós, Phys. Lett. B 438, 255 (1998).

[31] M. Quirós, talk given at the SUSY98 Conference, Oxford, England, 1998, http://hepnts.rl.ac.uk/SUSY98/.

[32] I. Antoniadis, S. Dimopoulos, A. Pomarol, and M. Quirós, Nucl. Phys. B544, 503 (1999).

[33] N.V. Krasnikov, Pis'ma Zh. Éksp. Teor. Fiz. 67, 727 (1998) [JETP Lett. 67, 766 (1998)].

[34] M. Sohnius, Phys. Rep. 128, 39 (1985).

[35] E. Sharpe, Nucl. Phys. B523, 211 (1998).

[36] G. Giudice and A. Pomarol, Phys. Lett. B 372, 253 (1996).

[37] C.P. Burgess, L.E. Ibáñez, and F. Quevedo, Phys. Lett. B 447, 257 (1999); I. Antoniadis, E. Dudas, and A. Sagnotti, Nucl. Phys. B544, 469 (1999); I. Antoniadis, G. D'appollonio, E. Dudas, and A. Sagnotti, "Partial breaking of supersymmetry, open strings and $M$-theory,' hep-th/9812118. 